\title{
A multidimensional latent class Rasch model for the assessment of the Health-related Quality of Life
}

\author{
Silvia Bacci*†, Francesco Bartolucci*ł
}

September 14, 2018

\begin{abstract}
This work is a temporary and partial version. The final and integrated version has been accepted as contributed chapter in Christensen K.B., Kreiner S., Mesbah M. (eds.), Rasch related models and methods for health science, Wiley-ISTE, ISBN: 978-1-84821-222-0, expected for December 2012.
\end{abstract}

\section{Introduction}

The World Health Organization [WHO 95] defined the Quality of Life (QoL) as:

"the individuals' perceptions of their position in life in the context of their culture and the value systems in which they live, and in relation to their goals, expectations, standards and concerns. It is a broad-ranging concept affected in a complex way by the persons' physical health, psychological state, level of independence, social relationships, personal beliefs, and their relationship to the salient features of their environment."

In a survey about quality of life, Fayers and Machin [FAY 00] defined the Health related Quality of Life (HrQoL) as:

"the way, which according to health of a person influences his/her capacity to lead on physical and social normal activities."

The "normality" of an activity is a variable concept, where it depends on the reference population. The use of indicators about HrQoL in clinical and epidemiological contexts is various: in primis these

\footnotetext{
*Dipartimento di Economia, Finanza e Statistica, Università di Perugia, Via Pascoli 20, 06123 Perugia, Italy

${ }^{\dagger}$ email: silvia.bacci@stat.unipg.it

‡email: bart@stat.unipg.it
} 
indicators give additional information to evaluate the effect of care therapies on a patient. More and more, HrQoL is considered as a secondary endpoint of clinical trials, because the main focus is on the survival. However, in some contexts, such as that of pain therapies for terminal cancer patients, it rises to a primary endpoint.

The main problem related to HrQoL concerns its measurement because this characteristic is not directly observable. Suitable measurement methods are then needed which are typically based on qualitative information, coming from ad hoc questionnaires, to be translated into quantitative information; see [MES 10] for a detailed illustration about statistical aspects involved in the measurement of HrQoL. In such a way it is possible to evaluate the patient's condition in relation to the general condition of the population so as to provide clinicians with information useful for the care and therapy decisional process. In this context, the Rasch model [RAS 61] represents an important tool to measure HrQoL. However, some important aspects must be taken into account.

First of all, HrQoL is a latent multidimensional concept, because its proper evaluation requires consideration of several dimensions (corresponding to different latent traits) that reflect individual health conditions and how well patients are coping with the stress due to illness. Often, two macrodimensions can be distinguished: a physical one and a psychological one; these are further separable in sub-dimensions, such as bodily pain and physical functioning in the former case, and mental health or vitality in the latter one. Usually, the latent traits corresponding to the different dimensions are highly correlated and are also correlated with other latent traits, such as those corresponding to some psychopathological disturbs, mainly anxiety and depression. However, the classic Rasch model is based on the assumption of unidimensionality. The easiest approach that is adopted when this assumption is not realistic consists of estimating separate Rasch models for subsets of items measuring different latent traits, but this method does not allow us to measure the correlation between these latent traits. A more suitable approach is based on in the multidimensional extension of Rasch models.

The second aspect is that, in many applications, it is of interest to detect homogeneous classes of individuals who have very similar latent characteristics. Detecting these classes of individuals can be, not only more realistic, but also more convenient for the decisional process because individuals in the same class will receive the same clinical treatment.

In order to analyse HrQoL data taking into account the above aspects, in this chapter we proposed the use of a version of the Rasch model which belongs to the class of multidimensional Item Response 
Theory (IRT) models proposed by Bartolucci [BAR 07]. The model is characterized by two main features: ( $i$ ) more latent traits are simultaneously considered (multidimensionality assumption); (ii) these latent traits are represented by a random vector having a discrete distribution common to all subjects (discreteness assumption). Each support point of this distribution identifies a different class of individuals. Obviously, these are latent classes, in the sense that we do not know to which class a given individual belongs; moreover, we do not know how many latent classes exist. The adopted model is then related to the latent class (LC) model (see [LAZ 68]; [GOO 74]) and for this reason will be referred to as the multidimensional LC Rasch model.

We note that the LC model originates as a method to classify individuals on the basis of categorical responses, but, more recently, the same discrete latent structure on which this model is based has been exploited to account for the unobserved heterogeneity between subjects into other models. Using this structure can be considered as an alternative to the inclusion of continuous random effects which avoids a parametric specification of the distribution of these effects. A semi-parametric model then results. In particular, this structure has been used by [LIN 91] and [FOR 95] to define a unidimensional LC Rasch model, which is a particular case of the model that we here adopt. An alternative generalization of the Rasch model to the LC analysis is represented by the mixture Rasch model of Rost [ROS 90]. It may be seen as an extension of LC Rasch model, which allows for different sets of item parameters for each latent class.

Another model which is strongly related to the multidimensional LC Rasch model is the LC factor model proposed by Magidson and Vermunt [MAG 01]. However, in the first approach each item response is affected by only one of the latent traits, and these latent traits may be correlated, whereas Magidson and Vermunt [MAG 01] assume that each item response may be simultaneously affected by two or more latent traits, which are mutually independent. We also have to mention alternative specifications of a multidimensional Rasch model that have been proposed; see, among others, the multidimensional marginally sufficient Rasch model proposed by Hardouin and Mesbah [HAR 04].

Aim of this chapter is also that of studying the correlation between the latent dimensions of HrQoL in cancer patients and those behind some psychopathological disturbs. This analysis involves two tests of dimensionality. The first is a likelihood ratio (LR) test which is based on the multidimensional LC Rasch model that exploits the discrete (or LC) marginal maximum likelihood (MML) approach. The second test is based on the Martin-Löf test (ML) approach [MAR 70] and exploits the conditional 
maximum likelihood (CML) estimation method (see also [GLA 95b]). Alternative tests have been proposed by several authors; for a review see Verhelst [VER 01]. In particular, we mention the approach of Christensen et al. [CHR 02], who propose a test similar to the first test we here use, but it is based on the assumption that the latent traits follow a multivariate normal distribution.

The remainder of this chapter is structured as follows. In Section 2 we describe the dataset used for the illustrative application. In Section 3 we present the multidimensional LC Rasch model of Bartolucci [BAR 07], with special attention to model assumptions and estimation from the practical point of view. In Section 4 we illustrate how to estimate the correlation between latent traits on the basis of the estimated parameters of this model. In the same section we describe the two tests of dimensionality based on the MML and CML approaches. Finally, in Section 5 we present the results of the application to the dataset described in Section 2.

\section{The dataset}

In order to illustrate the approach presented in this chapter, we analyze data which come from an Italian multi-centric clinical study. These data concern 275 oncological patients recruited from three different centres (Ancona, Perugia, and Messina). Patients were asked to fill some questionnaires about different latent characteristics; here, we consider HrQoL, anxiety, and depression. In particular, HrQoL is assessed by the "36-item Short-Form Health Survey" (SF-36) of Ware et al. [WAR 02], whereas anxiety and depression are assessed by the "Hospital Anxiety and Depression Scale" (HADS) of Zigmond and Snaith [ZIG 83]. The response rate is equal to 74\% (203 patients out of 275). However, the sample of respondents is here assumed to be representative of the entire sample, since there are no significant differences between the distributions of age, gender, marital status, education, and cancer diagnosis; see Table 1 for a comparison between the population and sample distributions of these variables.

SF-36 is a multidimensional test developed in the nineties to evaluate HrQoL during the last four weeks of illness; it has been validated in many different languages. The test consists of 36 polytomous items divided in the following 9 subsets (corresponding to different latent traits):

1. PF: physical functioning (10 items);

2. RF: role functioning, that is limitations in daily activities as a result due to physical health 
Table 1: Entire and respondent sample distributions of age, gender, marital status, education, and cancer diagnosis (column percentages).

\begin{tabular}{lcc}
\hline \hline & Entire sample & Respondents \\
\hline Age (years) & & \\
Mean & 54.6 & 54.3 \\
St. Dev. & 13.4 & 11.5 \\
\hline Gender (\%) & & \\
Female & 66.9 & 68.9 \\
Male & 33.1 & 31.1 \\
\hline Marital status (\%) & & \\
Single & 10.1 & 9.8 \\
Married & 79.4 & 80.3 \\
Separated & 4.2 & 3.3 \\
Widowed & 6.3 & 6.6 \\
\hline Education (\%) & & \\
Primary school & 12.6 & 12.9 \\
Middle school & 29.5 & 30.3 \\
High school & 38.8 & 37.6 \\
University & 19.1 & 19.1 \\
\hline Cancer diagnosis (\%) & & \\
Colon-rectum & 24.4 & 23.9 \\
Mammary & 45.6 & 46.7 \\
Uterine & 4.1 & 3.8 \\
Pulmonary & 8.8 & 8.7 \\
Prostate & 4.1 & 3.8 \\
Other & 13.0 & 13.0 \\
\hline Size & 275 & 203 \\
\hline
\end{tabular}

problems (4 items);

3. BP: bodily pain (2 items);

4. GH: general health (5 items);

5. VT: vitality (4 items);

6. SF: social functioning (2 items);

7. RE: role-emotional, that is limitations in daily activities as a result due to mental health problems (3 items);

8. MH: mental health (5 items);

9. HC: health change (1 item).

The items have a different number of response categories; to simplify the illustration of the results, in the present study all the items are dichotomized, with category 1 indicating the presence of a 
symptom or limitation (related to a low level of HrQoL), and category 0 indicating its absence (related to a high level of the HrQoL). Table 2 shows, for each item, which original responses correspond to new category 0 and which original responses correspond to new category 1. For the item coding used for the questionnaire SF-36 see [WAR 02].

Table 2: SF-36: original response categories and their dichotomization.

\begin{tabular}{lll}
\hline \hline \multirow{2}{*}{ Item* } & \multicolumn{2}{l}{ Dichotomized responses } \\
\cline { 2 - 3 } & 0 & 1 \\
\hline 1 & $1,2,3$ & 4,5 \\
2 & $1,2,3$ & 4,5 \\
$3 \mathrm{a}-3 \mathrm{l}$ & 3 & 1,2 \\
$4 \mathrm{a}-4 \mathrm{~d}$ & 2 & 1 \\
$5 \mathrm{a}-5 \mathrm{c}$ & 2 & 1 \\
6 & $1,2,3$ & 4,5 \\
7 & $1,2,3$ & $4,5,6$ \\
8 & $1,2,3$ & 4,5 \\
$9 \mathrm{a}, 9 \mathrm{~d}, 9 \mathrm{e}, 9 \mathrm{~h}$ & $1,2,3,4$ & 5,6 \\
$9 \mathrm{~b}, 9 \mathrm{c}, 9 \mathrm{f}, 9 \mathrm{~g}, 9 \mathrm{i}$ & 5,6 & $1,2,3,4$ \\
10 & $3,4,5$ & 1,2 \\
$11 \mathrm{a}, 11 \mathrm{c}$ & 4,5 & $1,2,3$ \\
11b, 11d & $1,2,3$ & 4,5 \\
\hline * Item labels are referred to the Italian \\
version of SF-36.
\end{tabular}

HADS is a questionnaire designed to assess anxiety and depression in patients with organic diseases, such as cancer. The questionnaire is composed by 14 items referred to two dimensions:

1. anxiety (7 items);

2. depression (7 items).

All items have four response categories but have been dichotomized for our analysis, with category 1 (corresponding to original categories 2 and 3) indicating the presence of anxiety (or depression), and category 0 (corresponding to original categories 0 and 1 ) indicating its absence. For the item coding used for the questionnaire HADS see [ZIG 83].

Note that, in both questionnaires, every item is assumed to measure only one latent trait, but these latent traits may be correlated. 


\section{The multidimensional latent class Rasch model}

In the following, we describe the model that we adopt for the analysis of HrQoL data. We recall that this is a version of the Rasch model which belongs the class of IRT models proposed in [BAR 07] and that the main differences with respect to the classic Rasch model [RAS 61] are that $(i)$ multidimensionality is assumed and (ii) the latent traits are assumed to have a discrete distribution. To facilitate the illustration of the model, we make explicit reference to the application described in Section 2.

\subsection{Model assumptions}

Let $n$ denote the number of subjects in the sample and suppose that they respond to $k$ test items which measure $D$ different latent traits or dimensions. Also let $\mathcal{I}_{d}, d=1, \ldots, D$, be the subset of $\mathcal{I}=\{1, \ldots, k\}$ containing the indices of the items measuring the latent trait of type $d$ and let $k_{d}$

denotes the cardinality of this subset, so that $k=\sum_{d=1}^{D} k_{d}$. Since we assume that every item measures only one latent trait, the subsets $\mathcal{I}_{d}$ are disjoint; obviously, these latent traits may be correlated. In our study, $n=203$ and $k=50$ (36 items from SF-36 and 14 items from HADS) and, on the basis of validation studies about these questionnaires, we know that the items measure $D=11$ different latent traits: 9 related to HrQoL and 2 related to psychopathological disturbs.

In this context, the classic Rasch model, based on the assumption

$$
\operatorname{logit}\left[p\left(X_{i}=1 \mid \Theta=\theta\right)\right]=\theta-\beta_{i}, \quad i=1, \ldots, k,
$$

where $\theta$ can be discrete or continuous, is extended in a multidimensional way on the basis of the following parameterization:

$$
\operatorname{logit}\left[p\left(X_{i}=1 \mid \boldsymbol{\Theta}=\boldsymbol{\theta}\right)\right]=\sum_{d=1}^{D}\left(\delta_{i d} \theta_{d}-\beta_{i}\right), \quad i=1, \ldots, k .
$$

In the above expressions, $X_{i}$ is the random variable corresponding to the response to item $i$; in particular, $X_{i}=1$ means the presence of the symptom or disturb detected by the item and $X_{i}=0$ means its absence. Moreover, in the unidimensional version, $\Theta$ is the latent random variable corresponding to the trait of the interest and $\theta$ denotes one of its possible values, whereas $\beta_{i}$ is the difficulty of item $i$. For the multidimensional version, $\boldsymbol{\Theta}=\left(\Theta_{1}, \ldots, \Theta_{D}\right)^{\prime}$ is the vector of latent variables corresponding to the different traits measured by the test items, $\boldsymbol{\theta}=\left(\theta_{1}, \ldots, \theta_{D}\right)^{\prime}$ denotes one of its possible real- 
izations, and $\delta_{i d}$ is a dummy variable equal to 1 if item $i$ belongs to $\mathcal{I}_{d}$ (and then it measures the $d$ th latent trait) and to 0 otherwise.

Note that, as already mentioned, the adopted model is based on a specific formulation of multidimensionality, in which each item measures only one latent trait; we are then assuming the so-called between-item multidimensionality. This is different from the so-called within-item multidimensionality, in which each item measures more than one latent trait (see Adams et al. [ADA 97] for a detailed description). Another important point is that the unidimensional formulation based on (1) is a special case of (2). In particular, one of the possible situations in which formulation (2) specialises into (1) is when the possible ability vectors $\boldsymbol{\theta}$ have elements equal each other.

In order to clarify the above point, consider a simple example involving only two latent traits, so that $\boldsymbol{\Theta}=\left(\Theta_{1}, \Theta_{2}\right)^{\prime}$, which may assume the values represented in Figure 1 .

(a) Unidimensional case

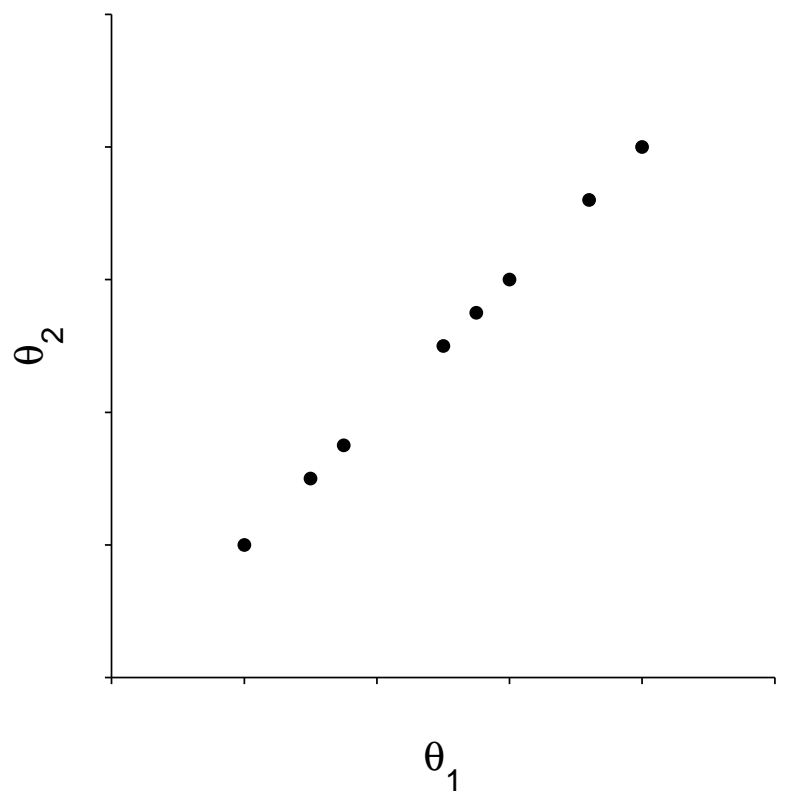

(b) Bidimensional case

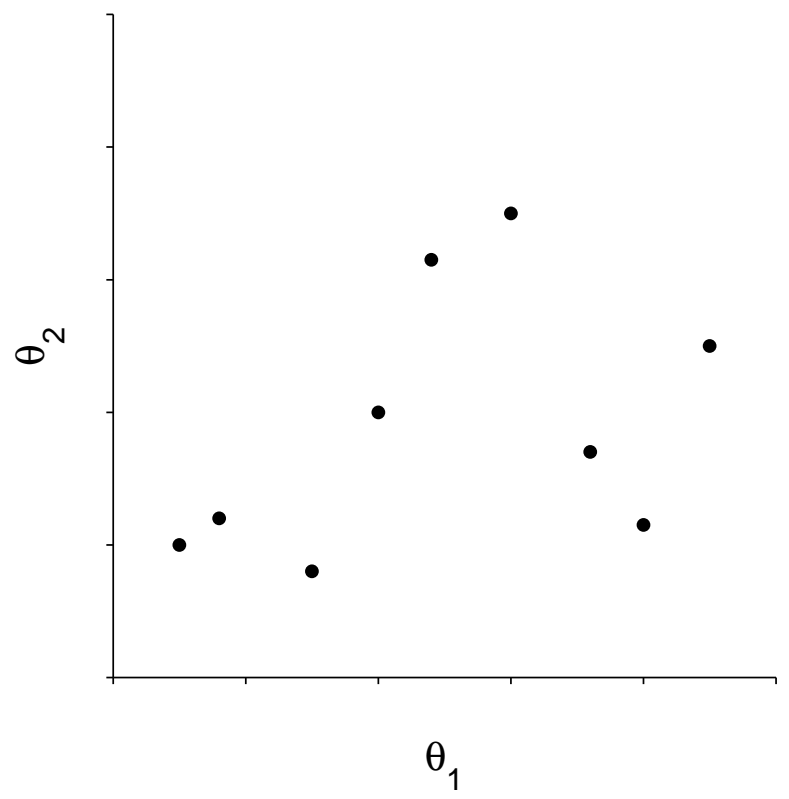

Figure 1: Plots of $\left(\theta_{1}, \theta_{2}\right)$ possible values.

We observe that panel $(a)$ corresponds to the situation of unidimensionality, because all points are aligned on the bisector. In other words, one latent trait coincides with the other and the actual number of dimensions is one. On the contrary, in panel $(b)$ the points are not on a bisector and there is not a complete ordering of them; in this case it is not possible to completely determine one latent trait from the other and we can consider the two latent traits as corresponding to two distinct dimensions.

In order to complete the model specification, we need to assume a distribution for the vector $\boldsymbol{\Theta}$, 
since we adopt a random-effects rather than a fixed-effects approach. In the latter, a distinct vector of parameters would be estimated for each subject, but this would not allow certain analyses which are of interest in the present context.

In a standard formulation, a continuous distribution $\pi(\boldsymbol{\theta})$, such as the normal distribution, is adopted. In such a case, the manifest distribution of the full response vector, $\mathbf{X}=\left(X_{1}, \ldots, X_{k}\right)^{\prime}$, is given by

$$
p(\mathbf{x})=p(\mathbf{X}=\mathbf{x})=\int_{\boldsymbol{\theta}} p(\mathbf{x} \mid \boldsymbol{\theta}) \pi(\boldsymbol{\theta}) d \boldsymbol{\theta},
$$

where, because of the assumption of local independence,

$$
p(\mathbf{x} \mid \boldsymbol{\theta})=p(\mathbf{X}=\mathbf{x} \mid \mathbf{\Theta}=\boldsymbol{\theta})=\prod_{i=1}^{k} p\left(X_{i}=x_{i} \mid \mathbf{\Theta}=\boldsymbol{\theta}\right) .
$$

Note that, because of the between-item multidimensionality assumption, we assume local independence separately for each dimension and therefore

$$
\prod_{i=1}^{k} p\left(X_{i}=x_{i} \mid \boldsymbol{\Theta}=\boldsymbol{\theta}\right)=\prod_{d=1}^{D} \prod_{h=1}^{k_{d}} p\left(X_{d h}=x_{d h} \mid \boldsymbol{\Theta}=\boldsymbol{\theta}\right)=\prod_{d=1}^{D} \prod_{h=1}^{k_{d}} p\left(X_{d h}=x_{d h} \mid \Theta_{d}=\theta_{d}\right),
$$

where $X_{d h}$ denotes the response variable for the $h$-th item measuring dimension $d$. Note that the notation $X_{d h}$ is equivalent to the previous one, in the sense that each $X_{i}$ coincides with a certain $X_{d h}$ for suitable $d$ and $h$. However, in this way we make explicit the dimension measured by this item.

It is worth recalling that the theory about the Rasch model does not require the latent trait of interest to have a continuous distribution. Moreover, assuming a discrete distribution may have some advantages. Among others, it can lead to more parsimonious models in given situations, it can be used to test the additivity assumption of the Rasch model, and to construct empirical Bayes estimators of abilities [LIN 91]. We adopted this alternative approach and, therefore, we assume that the random vector $\Theta$ has a discrete distribution with support $\left\{\boldsymbol{\zeta}_{1}, \ldots, \boldsymbol{\zeta}_{C}\right\}$. In other words, we assume that the population is composed by $C$ latent classes or subpopulations. We outline that the number of latent classes is the same for each dimension. In theory, it should also be possible to assume a more general model with a latent class structure that differs between dimensions, but in this case the interpretability of parameters is not straightforward. As for the conventional LC model (see [LAZ 68]; [GOO 74]), the manifest distribution of the full response vector is given by

$$
p(\mathbf{x})=p(\mathbf{X}=\mathbf{x})=\sum_{c=1}^{C} p\left(\mathbf{x} \mid \boldsymbol{\zeta}_{c}\right) \pi_{c}
$$


where $\pi_{c}=p\left(\boldsymbol{\Theta}=\boldsymbol{\zeta}_{c}\right)$ is the weight of the $c$ th latent class, that is the probability that a subject belongs to this latent class. Note that, according to equation $(4), p\left(\mathbf{x} \mid \boldsymbol{\zeta}_{c}\right)$ is defined as

$$
p\left(\mathbf{x} \mid \boldsymbol{\zeta}_{c}\right)=p\left(\boldsymbol{X}=\mathbf{x} \mid \boldsymbol{\Theta}=\boldsymbol{\zeta}_{c}\right)=\prod_{d=1}^{D} \prod_{h=1}^{k_{d}} p\left(X_{d h}=x_{d h} \mid \Theta_{d}=\zeta_{c d}\right), \quad c=1, \ldots, C .
$$

The specification of the multidimensional LC Rasch model, based on the assumptions illustrated above, univocally depends on the number of latent classes $(C)$ and on how items are associated to the different dimensions (i.e., the subsets $\mathcal{I}_{d}, d=1, \ldots, D$ ). Maximum likelihood estimation of this model is illustrated in the following.

\subsection{Maximum likelihood estimation and model selection}

Assuming that the subjects in the sample are independent, the log-likelihood of the model is

$$
\ell(\boldsymbol{\eta})=\sum_{\mathbf{x}} n(\mathbf{x}) \log [p(\mathbf{x})]
$$

where $\boldsymbol{\eta}$ is the vector containing all the parameters, $n(\mathbf{x})$ is the frequency of the response pattern $\mathbf{x}$, and the probability $p(\mathbf{x})$ is defined as in equation (6) depending on $\boldsymbol{\eta}$. Moreover, the sum $\sum_{\mathbf{x}}$ ranges over all the possible configurations of the response vector.

In order to maximize $\ell(\boldsymbol{\eta})$, and then obtaining the (discrete) MML estimate of $\boldsymbol{\eta}$, we make use of the EM algorithm [DEM 77], which is an iterative algorithm based on alternating two steps (E and $\mathrm{M})$. The expectation (E) step consists of computing the expected value of the complete log-likelihood evaluated at the current value of the parameters. The maximization (M) step consists of updating these parameters by maximizing the expected log-likelihood found at the E step. These parameters are then used to determine the distribution of the latent variables at the next E step. The two steps are performed until convergence. See [BAR 07] for a detailed description of this estimation algorithm and its initialization. At this stage we only stress that, in order to prevent the problem of the multimodality of the model likelihood, it is advisable to try different starting values chosen by both deterministic and random rules. We denote by $\hat{\boldsymbol{\eta}}$ the MML estimate of $\boldsymbol{\eta}$, that is the value that, at convergence of EM algorithm, corresponds to the highest log-likelihood. Similar notation is adopted for the single parameters.

Once the model is fitted, we can allocate each subject to one of the latent classes. The allocation depends on the specific item response pattern $\mathbf{x}$ provided by the subject. In particular, for each 
pattern $\mathbf{x}$ it is possible to estimate the posterior probabilities of belonging to latent class $c$ as follows

$$
\hat{p}\left(\boldsymbol{\zeta}_{c} \mid \mathbf{x}\right)=\hat{p}\left(\boldsymbol{\Theta}=\boldsymbol{\zeta}_{c} \mid \mathbf{X}=\mathbf{x}\right)=\frac{\hat{p}\left(\mathbf{x} \mid \boldsymbol{\zeta}_{c}\right) \hat{\pi}_{c}}{\sum_{h=1}^{C} \hat{p}\left(\mathbf{x} \mid \boldsymbol{\zeta}_{h}\right) \hat{\pi}_{h}}, \quad c=1, \ldots, C .
$$

An individual is assigned to the latent class with the highest posterior probability.

An important phase connected with the estimation process is represented by the model selection, with special attention to the choice of the number of latent classes $(C)$. A first approach [KIE 56] would suggest to use the value of $C$ corresponding to the saturation point beyond which the likelihood of the assumed model fails to increase. For the unidimensional LC Rasch model, the existence of this saturation point was established by Lindsay et al. [LIN 91]. However, in our context, this number of classes could be so large that the model becomes non-identifiable or almost non-identifiable. To avoid this, several authors suggest to use information criteria, which are typically based on penalizing the log-likelihood by a factor that takes into account the number of parameters as a measure of the model complexity.

One of the most known information criteria is represented by the Bayesian information criterion (BIC) of Schwarz [SCH 78], which is based on the index

$$
B I C=-2 \hat{\ell}+g \ln (n)
$$

where $\hat{\ell}$ is the maximum value of the log-likelihood and $g$ is the number of non-redundant parameters. For the multidimensional LC Rasch model, in particular, we have

$$
g=(C-1)+D C+(k-D)
$$

since there are $C-1$ mass probabilities for the classes, $D C$ ability parameters, and $k-D$ difficulty parameters (we have to consider that some of them are constrained to 0 in order to ensure model identifiability). Among different models, the one with the smallest value of the BIC index has to be preferred. For an illustration in the context of mixture models, which is strongly related to the present one, see McLachlan and Peel [MCL 00], Ch. 6.

\subsection{Software details}

In our application, we used the software accompanying the paper [BAR 07] to estimate the model and choose the number of classes. This software can be downloaded from the web page http://www.stat.unipg.it/bartolucci and is quite simple to use for practitioners. It is mainly based on a MATLAB function, named lcest.m, which requires the following inputs: 
- a matrix with each row corresponding to a different configuration of item responses and each column corresponding to an item (responses are coded as 0 and 1 and are suitably separated);

- a vector with the frequencies of the observed response configurations;

- the number of latent classes;

- the type of starting values (deterministic or random);

- the type of model (LC model, multidimensional LC Rasch model, multidimesional LC twoparameter logistic model);

- a matrix indicating the multidimensional structure of the set of items (any row contains the set of items referred to the same dimension).

The following output is obtained from lcest.m for the estimated model:

- maximum log-likelihood;

- estimated probabilities of the latent classes;

- $\mathrm{BIC}$ index;

- estimated difficulty parameters (and discriminating indices for the LC two-parameter logistic model);

- estimated support points for each latent class and for each latent dimension;

- estimated probability of responding 1 to every item for each latent class;

- estimated posterior probability of belonging to every latent class for each response configuration.

Function lcest.m also allows us to select the optimal number of latent classes. We have to estimate a multidimensional LC Rasch model with an increasing number of latent classes and the best model is chosen on the basis of a given selection criterion, such as BIC. In a similar way we can estimate the multidimensional LC Rasch model with different multidimensional structures in order to choose the optimal number of dimensions and to evaluate the possibility of collapsing two dimensions into a single one (see Section 4 for theoretical details on tests of dimensionality and Section 5 for an application). 
Other outputs, such as the correlations between latent dimensions or between every item and the corresponding dimension, cannot be obtained directly by lcest.m, but a little extra programming is sufficient.

\subsection{Concluding remarks about the model}

It is already clear that a crucial assumption of the multidimensional LC Rasch model described above is that of discreetness of the latent trait distribution. Alternatively, we could assume that the vector $\Theta$ follows a multivariate normal distribution of dimension $D$. However, if the variance-covariance matrix of this distribution is unconstrained, computing the manifest distribution of $\boldsymbol{X}$ would require complex numerical tools when $D$ is greater than 2 , as in our application, because a high dimensional integral is involved (see equation (3)). This has obvious implications on the parameter estimation and on the possibility of making a comparison with our model, which is based on a discrete latent trait distribution.

On the other hand, the model based on a multivariate normal distribution for the latent traits would be easily estimable under the constraint that the variance-covariance matrix is diagonal, so that the latent traits are independent. In fact, estimating this model is equivalent to estimating a separate unidimensional normal Rasch model for each dimension. The same holds for the version based on a discrete latent trait distribution. It is worth noting that, with the same number of latent classes $(C)$, the latter model involves more non-redundant parameters than the multidimensional LC Rasch model that we propose to use. In fact, this model has $D(C-1)+D C+(k-D)$ parameters which is larger than the number of parameters given in (10).

Though estimating separate unidimensional Rasch models does not allow us to study the correlation between the latent traits, this may be useful for assessing whether the assumption of discreteness of the latent trait distribution is suitable or not for the data at hand. This amount to comparing the global fit of these models under this assumption with that obtained under the assumption of normality. Such comparison may be based on standard criteria, such as BIC.

Finally, we have to remark that the multidimensional LC Rasch model may be easily extended to deal with polytomous items and incomplete data. Concerning the first issue, the extension would be based on adopting a suitable parameterization of the conditional response distribution given the latent traits, which extends that in (2). At this regard, we can use one of the parametrizations illustrated 
by Samejima [SAM 96] in her review of IRT models for polytomous data. About incomplete data, we have to clarify that the model and the estimation algorithm can be easily extended to treat the case of incomplete responses by design, because it is sufficient to drop, for each subject, the items to which the subject does not respond. The extension to the case of informative missing responses is not so obvious.

\section{Inference on the correlation between latent traits}

In our context, the main interest is on the study of the correlation between the latent traits corresponding to the different dimensions.

First of all, for two dimensions $d_{1}$ and $d_{2}$ (e.g., BP and SF), the correlation may be measured through the index

$$
\hat{\rho}_{d_{1} d_{2}}=\sum_{c=1}^{C} \hat{\zeta}_{c d_{1}}^{*} \hat{\zeta}_{c d_{2}}^{*} \hat{\pi}_{c}
$$

where $\hat{\zeta}_{c d}^{*}, c=1, \ldots, C, d=1, \ldots, D$, is the standardised estimate of the latent trait level referred to dimension $d$ for the subjects in latent class $c$. In practice, this is the $d$ th element of $\hat{\boldsymbol{\zeta}}_{c}$, once the average latent trait level has been subtracted from each element and this difference has been divided by the standard deviation. Moreover, $\hat{\pi}_{c}$ is the estimate of the weight of the $c$-th latent class.

A crucial point is how to test the hypothesis that two dimensions $d_{1}$ and $d_{2}$ are perfectly correlated. This hypothesis is strongly related to the hypothesis that the items in $\mathcal{I}_{d_{1}}$ and $\mathcal{I}_{d_{2}}$ measure the same latent trait. Given the parameterization in (2), the latter may be expressed as $H_{0}: \zeta_{c d_{2}}=\zeta_{c d_{1}}+a$, with $c=1, \ldots, C$, where $a$ is an arbitrary constant. In the following, we deal with two different approaches to test $H_{0}$.

The first approach is directly based on the LR statistic between the multidimensional latent class Rasch model, in which a separate latent variable is used to represent each dimension, and a restricted version of this model in which dimensions $d_{1}$ and $d_{2}$ are collapsed into a single one. Both models are estimated by the discrete MML method, and then by the EM algorithm illustrated at the end of Section 3. From this estimation we obtain the maximum log-likelihood of the general model $\left(\hat{\ell}_{1}\right)$ and that of the restricted model $\left(\hat{\ell}_{0}\right)$. It has to be clear that both models are based on the same number of latent classes; they only differ in the number of latent variables and in how items are allocated to these variables. On the basis of these likelihoods, we obtain the following LR test statistic for the null 
hypothesis $H_{0}$ :

$$
L R_{1}=-2\left(\hat{\ell}_{0}-\hat{\ell}_{1}\right)
$$

Under $H_{0}, L R_{1}$ has an asymptotic chi-square distribution with $C-1$ degrees of freedom, where $C$ is the selected number of latent classes. We reject $H_{0}$ for high values of $L R_{1}$, that is values larger than a suitable percentile of the asymptotic distribution; otherwise, we do not reject this hypothesis, implying that the two dimensions are indeed collapsible. In order to measure the evidence provided by the data in favour of $H_{0}$, we can also compute a $p$-value, as the value of the survival function of the asymptotic distribution at $L R_{1}$.

One of the main advantages of the above LR test is that, if properly extended, it can be also used when items discriminate differently among subjects (i.e., when the Rasch paradigm does not hold); however, this aspect is beyond the purposes of the present chapter. On the other hand, this approach requires the choice of the number of latent classes and the results may depend on this choice. For this reason we also consider a second approach for testing if two sets of items measure the same dimension, which does not require to formulate any assumption on the distribution of the latent variables. This approach is based on the ML test [GLA 95b]. See Martin-Löf [MAR 70] for the original version of the test for dichotomous items split in two dimensions and Christensen et al. [CHR 02] for a generalization to polytomous items and to situations with more than two dimensions. It is worth mentioning that, in the psychometric literature, alternative tests are available which may be more powerful in certain situations. We refer, in particular, to the class of one degree of freedom tests proposed by Verhelst [VER 01].

ML test is again an LR test between two models. The main difference with respect to the test based on statistic $L R_{1}$ is that the maximum log-likelihoods of the two models under comparison are obtained from the CML estimates (see [RAS 61]; [AND 70]; [AND 72]). The maximum log-likelihood of the general model obtained in this way is denoted by $\tilde{\ell}_{1}$, whereas that of the restricted model is denoted by $\tilde{\ell}_{0}$. The resulting LR test statistic is then

$$
L R_{2}=-2\left(\tilde{\ell}_{0}-\tilde{\ell}_{1}\right)
$$

In particular, the first log-likelihood is obtained as

$$
\tilde{\ell}_{1}=\tilde{\ell}_{1 c}^{(1)}+\tilde{\ell}_{1 c}^{(2)}+\tilde{\ell}_{1 m}
$$

where $\tilde{\ell}_{1 c}^{(1)}$ is the maximum conditional log-likelihood for the items in $\mathcal{I}_{d_{1}}, \tilde{\ell}_{1 c}^{(2)}$ is the maximum con- 
ditional $\log$-likelihood for the items in $\mathcal{I}_{d_{2}}$, both obtained through the CML method, and $\tilde{\ell}_{1 m}$ is the maximum marginal log-likelihood of the multinomial model for the distribution of the scores. The latter may be expressed as

$$
\tilde{\ell}_{1 m}=\sum_{r_{1}=0}^{k_{d_{1}}} \sum_{r_{2}=0}^{k_{d_{2}}} n_{r_{1} r_{2}} \log \left(n_{r_{1} r_{2}} / n\right),
$$

where $r_{1}$ is the test score for the items in the subset $\mathcal{I}_{d_{1}}$ (e.g., PF), $r_{2}$ is the test score for the items in the subset $\mathcal{I}_{d_{2}}$ (e.g., RF), and $n_{r_{1} r_{2}}$ is the frequency of subjects with scores $r_{1}$ and $r_{2}$ at the two subsets; see also [VER 01]. We recall that the test score is the number of items that are responded by category 1 and that $k_{d_{1}}$ and $k_{d_{2}}$ are the number of items from the first dimension (e.g., PF) and from the second dimension (e.g., RF), respectively.

A decomposition similar to that in (14) holds for $\tilde{\ell}_{0}$. We have

$$
\tilde{\ell}_{0}=\tilde{\ell}_{0 c}+\tilde{\ell}_{0 m}
$$

where $\tilde{\ell}_{0 c}$ is the maximum conditional log-likelihood for the items in $\mathcal{I}_{d_{1}} \cup \mathcal{I}_{d_{2}}$, and

$$
\tilde{\ell}_{0 m}=\sum_{r=0}^{k_{d_{1}}+k_{d_{2}}} n_{r} \log \left(n_{r} / n\right) .
$$

In the above expression, $r$ is the test score achieved at the items in both subsets, the frequency of which is denoted by $n_{r}$. Under $H_{0}$, the test-statistic $L R_{2}$ has an asymptotic chi-square distribution with $k_{d_{1}} k_{d_{2}}-1$ degrees of freedom.

Though the ML test has the advantage of not requiring the choice of the number of latent classes neither the formulation of distribution assumptions on the latent variables, Verhelst [VER 01] and Christensen et al. [CHR 02] found that its power is significantly affected by the length of the questionnaire and it may be disappointingly low with strongly correlated latent dimensions. More precisely, it can be verified that the null distribution may deviate from the asymptotic chi-square distribution because of the large number of items in comparison with the sample size. In the psychometric literature there are several proposals to solve this problem. For example, Christensen and Kreiner [CHR 07] proposed a Monte Carlo test procedure for computing $p$-values. Moreover, another problem connected with the ML test concerns the fact that this test can only be used when the Rasch paradigm holds, since otherwise the CML method cannot be applied.

In order to take into account advantages and disadvantages of the two tests described in this section, in our empirical study we apply both. The LR test based on the discrete MML is calculated by means of a set of MATLAB functions which are available upon request. The ML test is performed 
by means of STATA as concerning the computation of the maximum conditional log-likelihoods $\left(\tilde{\ell}_{0 c}\right.$,

$\left.\tilde{\ell}_{1 c}^{(1)}, \tilde{\ell}_{1 c}^{(2)}\right)$, and through a suitable MATLAB function as concerning the marginal counterparts $\left(\tilde{\ell}_{0 m}\right.$, $\left.\tilde{\ell}_{1 m}\right)$. The same methods could be applied by alternative statistical softwares, such as MULTIRA of Carstensen and Rost [CAR 01], the \%pml SAS macro of Christensen and Bjorner [CHR 03] or DIGRAM of Kreiner [KRE 03].

Finally, we have to underline that the above methodology gives valid results provided that the Rasch paradigm holds for each dimension. In order to check that this is the case, we have to fit separate Rasch models for each of these dimensions, and those that violate this paradigm should not be included in the analysis. For this purpose, several testing procedures are available in the literature (see, among others, [LIN 94] and [GLA 95a]). These procedures are mainly based on the comparison with the two-parameter logistic model and with the saturated model by chi-square or LR statistics, and on infit and outfit statistics.

\section{Application results}

First of all, with reference to the data described in Section 2, we verified that the Rasch paradigm clearly holds for only five dimensions; we recall that each dimension corresponds to a subset of items measuring a specific aspect and these subsets are defined by the structure of the questionnaires. Three of these dimensions are referred to HrQoL, that is BP, SF, and VT, whereas the other two are referred to psychopathological disturbs, that is anxiety and depression. Therefore, we based our analysis on the responses to the items referred to these five dimensions, whereas the items referred to the other ones were discarded. Overall, the data we analysed concern 22 items.

The first step of the analysis was focused on verifying if a discrete latent structure, on which the LC Rasch model is based, is indeed more suitable for the data at hand with respect to a continuous latent structure based on the normal distribution. As explained in Section 3.4, we cannot directly compare the multidimensional LC Rasch model with its normal counterpart because of the difficulties in estimating the latter. However, we can indirectly perform this comparison by estimating separate undimensional (LC and normal) Rasch models for each dimension. The results of this comparison are reported in Table 3 in terms of maximum log-likelihood and BIC index for each model.

We observe that the assumption that the distribution of the latent traits is discrete has to be preferred to the assumption that this distribution is normal. In fact, for each dimension, the unidimen- 
Table 3: Maximum log-likelihood and BIC index for the unidimensional Rasch model fitted for each dimension by the $M M L$ method under the assumption that the latent trait has a normal distribution and under the assumption that is has discrete distribution with a number of support points (latent classes) between 1 and \%; for each dimension, in bold are the data referred to the model with the smallest BIC.

\begin{tabular}{|c|c|c|c|c|c|c|c|c|c|}
\hline & & normal & & LC Rasc & model - & lumber of & latent $\mathrm{cl}$ & $\operatorname{sses}(C)$ & \\
\hline Dimensi & & Rasch model & 1 & 2 & 3 & 4 & 5 & 6 & 7 \\
\hline $\mathrm{BP}$ & $\hat{\ell}$ & -193.4 & -206.9 & -190.5 & -190.5 & -190.5 & -190.5 & -190.5 & -190.5 \\
\hline & $B I C$ & 402.7 & 424.4 & 402.2 & 412.8 & 423.5 & 434.1 & 444.8 & 455.4 \\
\hline $\mathrm{SF}$ & $\hat{\ell}$ & -157.8 & -163.1 & -147.2 & -147.2 & -147.2 & -147.2 & -147.2 & -147.2 \\
\hline & $B I C$ & 331.5 & 336.9 & 315.7 & 326.4 & 337.0 & 347.6 & 358.3 & 368.9 \\
\hline VT & $\hat{\ell}$ & -495.1 & -511.0 & -454.6 & -446.8 & -446.8 & -446.8 & -446.8 & -446.8 \\
\hline & $B I C$ & 1016.7 & 1043.2 & 941.2 & 936.2 & 946.8 & 957.5 & 968.1 & 978.8 \\
\hline Anxiety & $\hat{\ell}$ & -671.6 & -753.5 & -653.4 & -642.7 & -641.0 & -641.0 & -641.0 & -641.0 \\
\hline & $B I C$ & 1385.8 & 1544.3 & 1354.8 & 1343.9 & 1351.1 & 1361.8 & 1372.4 & 1383.0 \\
\hline Depression & $\hat{\ell}$ & -636.5 & -701.6 & -607.7 & -602.0 & -602.0 & -602.0 & -602.0 & -602.0 \\
\hline & $B I C$ & 1315.5 & 1440.4 & 1263.2 & 1262.4 & 1273.0 & 1283.6 & 1294.2 & 1304.9 \\
\hline Overall & $\hat{\ell}$ & -2154.4 & -2336.1 & -2053.5 & -2029.2 & -2027.5 & -2027.5 & -2027.5 & -2027.5 \\
\hline & $B I C$ & 4452.2 & 4789.2 & 4277.1 & 4281.7 & 4331.4 & 4384.7 & 4437.7 & 4491.0 \\
\hline
\end{tabular}

sional LC Rasch model always attains a higher maximum log-likelihood than its normal counterpart, while the minimum BIC index is always smaller. The same happens at global level (see the last two rows of Table 3 ).

Then, we fitted the multidimensional LC Rasch model with an increasing number of latent classes. To this regard, in Table 4 we report the values of the maximum log-likelihood and of the BIC index obtained with a number of latent classes $(C)$ from 1 to 7 .

Table 4: Maximum log-likelihood and BIC index for the multidimensional latent class Rasch model with a number of latent classes between 1 and 7 ; in bold are the data referred to the model with the smallest $B I C$.

\begin{tabular}{lrrrrrrr}
\hline \hline & \multicolumn{7}{c}{ Number of latent classes $(C)$} \\
\cline { 2 - 8 } & \multicolumn{1}{c}{2} & \multicolumn{1}{c}{3} & \multicolumn{1}{c}{4} & \multicolumn{1}{c}{5} & \multicolumn{1}{c}{6} & \multicolumn{1}{c}{7} \\
\hline$\hat{\ell}$ & -2321.8 & -2066.7 & -2016.3 & -1989.8 & -1966.9 & $\mathbf{- 1 9 4 9 . 3}$ & -1937.7 \\
$B I C$ & 4760.6 & 4282.1 & 4213.2 & 4192.1 & 4178.3 & $\mathbf{4 1 7 5 . 0}$ & 4183.7 \\
\hline
\end{tabular}

According to the adopted selection criterion, we chose $C=6$ latent classes corresponding to $B I C=4,175.0$. We observe that this model has a better fit than the model which is equivalent to 
separate unidimensional LC Rasch models. For the latter we had 4,277.1 as minimum value of the BIC index; this value is reached with 2 latent classes (see Table 3). For the multidimensional LC Rasch model with $C=6$ latent classes, we obtained the parameter estimates (support points and class weights) displayed in Table 5. In particular, we estimated 5 support points (corresponding to the number of dimensions) for each of the 6 latent classes. Due to the adopted parameterization, see equation (2), very low values of these support points correspond to a negligible probability of response 1 , whereas very high values correspond to a negligible probability of response 0 . To facilitate the interpretation of the results, the latent classes are ordered on the basis of the conditional probability of response 1 for the first dimension (BP).

Table 5: Estimated support points and probabilities of the latent classes (for each dimension, in bold the highest support point, in italic the smallest one).

\begin{tabular}{lcccccr}
\hline \hline & \multicolumn{7}{c}{ Latent class } \\
\cline { 2 - 7 } Dimension & 1 & \multicolumn{1}{c}{3} & \multicolumn{1}{c}{4} & \multicolumn{1}{c}{5} & \multicolumn{1}{c}{6} \\
\hline BP & $-\infty$ & -2.17 & -0.92 & -0.91 & -0.20 & $\mathbf{1 . 4 8}$ \\
SF & -2.56 & $-\infty$ & -1.71 & -2.92 & $-\infty$ & $\mathbf{0 . 7 9}$ \\
VT & -2.36 & -3.28 & -0.46 & $\mathbf{1 . 0 9}$ & -2.23 & 0.93 \\
Anxiety & $\mathbf{0 . 0 0}$ & -4.87 & -3.40 & -1.85 & -1.55 & -0.05 \\
Depression & $\mathbf{1 . 3 0}$ & -4.10 & -1.99 & 0.12 & -1.50 & 0.76 \\
\hline Probability & 0.042 & 0.223 & 0.383 & 0.134 & 0.131 & 0.086 \\
\hline
\end{tabular}

A crucial point of the present study is the interpretation of the latent classes, which define homogeneous groups in the population. This interpretation is not always easy, but some useful suggestions come from the estimates of the support points and class probabilities. In particular, the smallest classes are the first ( $4.2 \%$ of subjects) and the last ( $8.6 \%$ of subjects). These classes contain, respectively, patients with the highest tendency to psychopathological disturbs and with the highest impairment with respect to $\mathrm{BP}$ and $\mathrm{SF}$. The second class contains more subjects (22.3\%); these subjects are in the best conditions with respect all dimensions, with the exception of BP. The third class is the largest (38.3\% subjects) and includes subjects with an intermediate tendency to be ill with respect to each dimension. Finally, the fourth and fifth latent classes have a very similar size $(13.4 \%$ and $13.1 \%$ of subjects) and include, respectively, subjects with the worst conditions with respect to VT and the best conditions with respect to SF. Note that individuals belonging to the second and the fifth latent class show a very good level with respect to SF.

As already mentioned, it is important to analyze the correlation between the different dimensions 
of HrQoL, and the correlation with anxiety and depression. At this aim, in Table 6 we report the correlation coefficients estimated on the basis of equation (11) for every pair of dimensions.

Table 6: Estimated correlation matrix between latent traits.

\begin{tabular}{lcccc}
\hline & SF & VT & Anxiety & Depression \\
\hline BP & 0.679 & 0.794 & 0.932 & 0.989 \\
SF & & 0.934 & 0.388 & 0.596 \\
VT & & & 0.530 & 0.707 \\
Anxiety & & & & 0.971 \\
\hline
\end{tabular}

As regards the dimensions of HrQoL, we observe that VT is strongly correlated especially with the other psychological dimension SF $(\hat{\rho}=0.934)$. As regards the association between the dimensions of HrQoL measured by the SF-36 questionnaire and the psychopathological disorders measured by the HADS questionnaire, both anxiety and depression show a very high correlation with BP $(\hat{\rho}=0.932$ and $\hat{\rho}=0.989$, respectively). On the other hand, the association of anxiety with SF and VT is much weaker $(\hat{\rho}=0.388$ and $\hat{\rho}=0.530$, respectively), and the association of depression with SF and VT assumes intermediate values $(\hat{\rho}=0.596$ and $\hat{\rho}=0.707$, respectively). Finally, as we may expect, the correlation between anxiety and depression is very high $(\hat{\rho}=0.971)$.

When the correlation between two latent traits is high, it is interesting to verify if the corresponding items actually measure the same dimension. At this aim, we performed both tests described in the previous section, that is the LR tests based on the discrete MML and the CML methods, for every pair of dimensions. LR statistics and the corresponding $p$-values are shown in Table 7 for the first test and in Table 8 for the second one.

Table 7: Results from the test of dimensionality based on the discrete MML parameter estimates; in brackets the p-values.

\begin{tabular}{lcccc}
\hline \hline & SF & VT & Anxiety & Depression \\
\hline BP & $27.1(0.000)$ & $14.0(0.007)$ & $22.2(0.000)$ & $27.5(0.000)$ \\
SF & & $12.5(0.014)$ & $32.1(0.000)$ & $26.3(0.000)$ \\
VT & & & $51.2(0.000)$ & $70.3(0.000)$ \\
Anxiety & & & & $9.1(0.059)$ \\
\hline
\end{tabular}

We observe that the values of the test statistic based on discrete MML method are almost always smaller than the corresponding values computed on the basis of the CML method, but the main conclusions are similar. In fact, both test statistics have a null asymptotic chi-square distribution, 
Table 8: Results from the test of dimensionality based on the CML parameter estimates (ML test); in brackets the p-values.

\begin{tabular}{lccrr}
\hline \hline & SF & VT & Anxiety & \multicolumn{1}{c}{ Depression } \\
\hline BP & $23.0(0.000)$ & $38.6(0.000)$ & $53.7(0.000)$ & $57.0(0.000)$ \\
SF & & $30.7(0.000)$ & $42.9(0.000)$ & $36.3(0.001)$ \\
VT & & & $126.7(0.000)$ & $112.7(0.000)$ \\
Anxiety & & & & $83.3(0.001)$ \\
\hline
\end{tabular}

but the second is based on a larger number of degree of freedom. Nevertheless, both kinds of test do not show any evidence of unidimensionality. A weak evidence of unidimensionality appears under the test based on the discrete MML estimates for anxiety and depression ( $p$-value equal to 0.059) and for VT and SF ( $p$-value equal to 0.014). Then, the present study confirms that the dimensions defined by the SF-36 and HADS questionnaires are indeed separate, and collapsing some of them would imply an inappropriate simplification of the phenomena investigated by the test items.

\section{Acknowledgements}

The authors are grateful to A. Bonacchi of University of Florence (IT) for making available the data. F. Bartolucci acknowledges the financial support of PRIN07 (grant 2007XECZ7L003) and of the "Einaudi Institute for Economics and Finance", Rome, IT.

\section{References}

[AND 70] ANDERSEN E.B., Asymptotic properties of conditional maximum likelihood estimators, Journal of the Royal Statistical Society, Series B, vol. 32, 1970, p. 283-301.

[AND 72] ANDERSEN E.B., The numerical solution of a set of conditional estimation equations, Journal of the Royal Statistical Society, Series B, vol. 34, 1972, p. 42-54.

[ADA 97] ADAMS R.J., WILSON M., WANG W.C., The multidimensional random coefficients multinomial logit model, Applied Psychological Measurement, vol. 21, 1997, 1-23.

[BAR 07] BARTOLUCCI F., A class of multidimensional IRT models for testing unidimensionality and clustering items, Psychometrika, vol. 72, 2007, p. 141-157.

[CAR 01] CARSTENSEN C.H., ROST J., MULTIRA (version 1.65) [Computer software and manual], 2001, retrieved from http://www.multira.de..

[CHR 02] CHRISTENSEN K.B., BJORNER J.B., KREINER S., PETERSEN J.H., Testing unidimensionality in polytomous Rasch models. Psychometrika, vol. 67 no.4, 2002, p. 563-574. 
[CHR 03] CHRISTENSEN K.B., BJORNER J.B., SAS macros for Rasch based latent variable modelling (Res. Rep. No. 03/13. Department of Biostatistics. University of Copenhagen, 2003.

Retrieved from http://www.ifsv.ku.dk/afdelinger/biostatistik/lokale/sasmacros.

[CHR 07] CHRISTENSEN K.B., KREINER S., A Monte Carlo approach to unidimensionality testing in polytomous Rasch models. Applied Psychological Measurement, vol. 31, 2007, p. 20-30.

[DEM 77] DEMPSTER A.P., LAIRD N.M., RUBIN D.B., Maximum likelihood from incomplete data via the EM algorithm (with discussion). Journal of the Royal Statistical Society, Series B, vol. 39, 1977, p. 1-38. [FAY 00] FAYERS P.M., MACHIN D., Quality of life. Assessment, analysis and interpretation, Wiley, 2000.

[FOR 95] FORMANN A.K., Linear logistic latent class analysis and the Rasch model, in G.H. Fischer, and I.W.Molenaar (Eds.), Rasch models: Foundations, recent developments, and applications, New York, Springer-Verlag, 1995, p. 239-255.

[GLA 95a] GLAS C.A.W., VERHELST N.D. Testing the Rasch model, in G.H. Fischer, and I.W. Molenaar (Eds.), Rasch models: Foundations, recent developments, and applications, New York, Springer-Verlag, 1995, p. 69-95.

[GLA 95b] GLAS C.A.W., VERHELST N.D. Tests of fit for polytomous Rasch models, in G.H. Fischer, and I.W. Molenaar (Eds.), Rasch models: Foundations, recent developments, and applications, New York, Springer-Verlag, 1995, p. 325-352.

[GOO 74] GOODMAN L.A., Exploratory latent structure analysis using both identifiable and unidentifiable models. Biometrika, vol. 61, 1974, p. 215-231.

[HAR 04] HARDOUIN J.B., MESBAH M., Clustering binary variables in subscales using an extended Rasch model and Akaike information criterion. Communication in Statistics. Theory and Methods, vol. 33, 2004, p. 1277-1294.

[KIE 56] KIEFER J., WOLFOWITZ J., Consistency of the maximum likelihood estimator in the presence of infinitely many incidental parameters. Annals of Mathematical Statistics, vol. 27, 1956, p. 887-906.

[KRE 03] KREINER S., Introduction to DIGRAM, Res.Rep.No. 03/10, Department of Biostatistics. University of Copenhagen, 2003.

[LAZ 68] LAZARSFELD P.F., HENRY N.W., Latent structure analysis, Boston, Houghton Mifflin, 1968.

[LIN 94] LINACRE, J. M., and B. D. WRIGHT. 1994. (Dichotomous mean-square) infit and outfit chi-square fit statistics. Rasch Measurement Transactions 8(2): 360.

[LIN 91] LINDSAY B., CLOGG C., GREGO J., Semiparametric estimation in the Rasch model and related exponential response models, including a simple latent class model for item analysis. Journal of the American Statistical Association, vol. 86, 1991, p. 96-107. 
[MAG 01] MAGIDSON J., VERMUNT J.K., Latent class factor and cluster models, bi-plots, and related graphical displays. Sociological Methodology, vol. 31, 2001, p. 223-264.

[MCL 00] MCLACHLAN G. J., PEEL, D., Finite Mixture Models, Wiley, 2000.

[MAR 70] MARTIN-LÖF P., Statistika modeller (Statistical Models): Anteckningar frän seminarier lasảret 1969-1970 (Notes from seminars in the academic year 1969-1970), with the assistance of Rolf Sundberg. Stockholm: Institütet för Försäkringsmatemetik och Matematiks Statistiks vid Stockholms Universitet, 1970.

[MES 10] MESBAH M., Statistical Quality of Life, in N. Balakrishnan (Ed.), Methods and Applications of Statistics in Life and Health Sciences, New York, Wiley, 2010, p. 839-864.

[RAS 61] RASCH G., On general laws and the meaning of measurement in psychology, Proceedings of the IV Berkeley Symposium on Mathematical Statistics and Probability, vol. 4, 1961, p. 321-333.

[ROS 90] ROST J., Rasch models in latent classes: an integration of two approaches to item analysis, Applied Psychological Measurement, vol. 14, 1990, p. 271-282.

[SAM 96] SAMEJIMA F., Evaluation of mathematical models for ordered polychotomous responses. Behaviormetrika, vol. 23, 1996, p. 17-35.

[SCH 78] SCHWARZ G., Estimating the dimension of a model. Annals of Statistics, vol. 6, 1978, p. 461-464.

[WAR 02] WARE J.E., KOSINSKI M., DEWEY J.E., How to Score Version 2 of the SF-36 Health Survey (Standard $\& 3$ acute forms). Lincoln RI, Quality Metric Incorporated, 2002.

[VER 01] VERHELST, N.D., Testing the unidimensionality assumption of the Rasch model, Methods of Psychological Research Online, vol. 6, 2001, p. 231-271.

[WHO 95] The WHOQOL Group, The World Health Organization Quality of Life Assessment (WHOQOL): position paper from the World Health Organization, Special Issue on Health-Related Quality of Life: what is it and how should we measure it? Social Science and Medicine, vol. 41(10), 1995, p. 1403-1409.

[ZIG 83] ZIGMOND A.S., SNAITH R.P., The hospital anxiety and depression scale, Acta Psychiatrika Scandinavica, vol. 67 no. 6,1983 p. 361-370. 